\title{
Spatial Correction Improves Accuracy of Catheter Positioning During Ablation of Premature Ventricular Contractions: Differences Between Ventricular Outflow Tracts And Other Localizations.
}

\section{Moritz Nies ( $\nabla$ m.nies@uke.de)}

University Heart and Vascular Center, Department of Cardiology, Hamburg https://orcid.org/0000-00029384-4151

\section{Ruben Schleberger}

University Heart and Vascular Center, Department of Cardiology, Hamburg

\section{Leon Dinshaw}

University Heart and Vascular Center, Department of Cardiology, Hamburg

\section{Niklas Klatt}

Schön Klinik Neustadt: Schon Klinik Neustadt, Department of Cardiology, Neustadt in Holstein

\section{Paula Muenkler}

University Heart and Vascular Center, Department of Cardiology, Hamburg

\section{Christiane Jungen}

University Heart and Vascular Center, Department of Cardiology, Hamburg

\section{Laura Rottner}

University Heart and Vascular Center, Department of Cardiology, Hamburg

\section{Marc D Lemoine}

University Heart and Vascular Center, Department of Cardiology, Hamburg

\section{Bruno Reißmann}

University Heart and Vascular Center, Department of Cardiology, Hamburg

\section{Andreas Rillig}

University Heart and Vascular Center, Department of Cardiology, Hamburg

Andreas Metzner

University Heart and Vascular Center, Department of Cardiology, Hamburg

Paulus Kirchhof

University Heart and Vascular Center, Department of Cardiology, Hamburg

\section{Christian Meyer}

EVK Düsseldorf, Division of Cardiology, Düsseldorf

\section{Research}


Keywords: hybrid mapping, premature ventricular contractions, activation mapping, catheter ablation

Posted Date: June 16th, 2021

DOI: https://doi.org/10.21203/rs.3.rs-594511/v1

License: (c) (1) This work is licensed under a Creative Commons Attribution 4.0 International License. Read Full License 


\section{Abstract}

\subsection{Purpose}

Hybrid activation mapping is a novel tool to correct for spatial displacement of the mapping catheter due to asymmetrical contraction of myocardium during premature ventricular contractions (PVC). The aim of this study is to describe the extent and cause of spatial displacement during PVC mapping and options for correction using hybrid activation mapping.

\subsection{Methods and Results}

We analyzed 5798 hybrid mapping points in 40 acquired hybrid maps of 22 consecutive patients (age $63 \pm 16$ years, $45 \%$ female) treated for premature ventricular contractions (PVCs). Median PVC-coupling interval was $552 \mathrm{~ms}$ (IQR $83 \mathrm{~ms}$ ). Spatial displacement was determined by measuring the dislocation of the catheter tip during PVC compared to the preceding sinus beat. Mean spatial displacement was $3.8 \pm 1.5 \mathrm{~mm}$ for all maps. The displacement was $1.3 \pm 0.4 \mathrm{~mm}$ larger for PVCs with non-outflow-tract origin compared to PVCs originating from the ventricular outflow tracts (RVOT/LVOT; $p=0.028$ ). Demographic parameters, PVC-coupling-interval and chamber of origin had no significant influence on the extent of spatial displacement.

\subsection{Conclusion}

Ectopic activation of the ventricular myocardium during PVCs results in spatial displacement of mapping points that is significantly larger for PVCs with non-outflow-tract origin. The correction for spatial displacement may improve accuracy of radiofrequency current (RFC)-application in catheter ablation of PVCs.

\section{Introduction}

During the last decades, catheter ablation of premature ventricular contractions (PVCs) has developed into a standard treatment for symptomatic patients [1-4]. Technical and procedural advances have improved outcome and safety so far that catheter ablation is now recommended as primary treatment option for outflow tract PVCs [5]. In light of these developments, the role of catheter ablation in clinical practice is expected to increase in the near future.

To guide the catheter for mapping and ablation, three-dimensional, electroanatomical mapping of PVCs is a routine method during interventional treatment [6].

During PVCs, the ectopic origin of myocardial activation results in an asymmetrical contraction sequence. This leads to a spatial shift of myocardial tissue during PVC compared to its location during normal sinus rhythm, as it has first been described by Andreu et al. [7]. Since catheter ablation is usually performed during sinus rhythm, this phenomenon can lead to imprecise localization of ablation targets. 
Correcting for this shift may facilitate the precise localization of the origin of PVCs and therefore allow to direct radio-frequency-current (RFC)-impulses with higher accuracy.

A novel mapping software tool (CARTO III, Software Version 7 Carto Prime, Biosense Webster) integrates an algorithm for correction of the aforementioned shift: for each registered PVC, the electrogram during the ectopic beat is paired with the location of the preceding sinus beat. This novel mapping algorithm is referred to as "hybrid mapping" [8]. Recently, first clinical results have been published for mapping and ablation using hybrid mapping $[8,9]$. However, the extent and influencing factors of spatial displacement remain unclear. Patient-specific factors and anatomical characteristics of different myocardial areas might affect spatial displacement. The aim of this study is to describe the extent and influencing factors of spatial displacement in hybrid activation mapping of PVCs.

\section{Materials And Methods}

\subsection{Patient selection}

For this study, all patients who underwent catheter ablation for PVC between April 2019 and April 2020 using the novel mapping feature were analyzed. Procedures, in which pacemapping was used due to insufficient intraprocedural incidence of PVCs, were excluded. Clinical characteristics were obtained by review of the medical records and charts. Structural heart disease was defined as coronary artery disease leading to interventional treatment, history of myocarditis, significant valvular disease leading to ventricular dysfunction, dilated/hypertrophic cardiomyopathy or systemic disease with cardiac manifestation (e.g. sarcoidosis).

\subsection{Mapping and ablation}

Ablation procedures were performed under conscious sedation using propofol and fentanyl. Orciprenaline was administered to provoke PVCs during ablation procedures when required. Catheters were positioned via femoral venous and/or retrograde arterial access depending on the respective chamber of interest. Systemic heparinization to achieve an activated clotting time of 250-300 seconds was performed for left-sided procedures.

Three-dimensional mapping was performed using CARTO III (Software Version 7 Carto Prime, Biosense Webster, Diamond Bar, CA, USA) with the integrated hybrid mapping module: Both the clinical PVC and a normal sinus beat were saved as ECG-pattern. When a PVC matching the predefined pattern was recorded, the local activation time (LAT) during the PVC was projected onto the catheter position recorded during the preceding sinus beat. In that way, catheter movement due to unphysiological contraction is corrected and the mapping point represents the position of the corresponding myocardium in sinus rhythm. The distance between catheter location during PVC and the location during the preceding sinus beat was defined as spatial displacement. The threshold for matching a PVC to the predefined pattern was set to $98 \%$. As reference for local activation time, a precordial lead with a well-defined, stable R-peak during PVCs was selected. Standard catheter for mapping and ablation was a $3.5 \mathrm{~mm}$ irrigated tip 
catheter (Carto NaviStar ThermoCool, 8 French, D-Curve, Biosense Webster). Ablation was performed in the area of earliest activation using radiofrequency current with a power between 20 and $50 \mathrm{~W}$ depending on the target area.

\subsection{Quantification of spatial displacement}

During the ablation procedure, we carefully reviewed the annotations to obtain correct data for spatial displacement. Offline, all non-hybrid points and floating points were deleted in order to create a map comprised exclusively of hybrid points. For each point, spatial displacement and PVC-coupling interval were analyzed. Additionally, the mean spatial displacement was calculated for all recorded hybrid points. In each map, the exact location of PVC-origin, chamber of PVC-origin, number of mapping points, number of hybrid mapping points, median spatial displacement and median PVC-coupling interval were analyzed.

\subsection{Statistical Analysis}

Descriptive statistics are presented as count and percentage for categorical and ordinal variables and as mean \pm standard deviation for continuous variables if normally distributed, and as median (interquartile range) otherwise.

To analyze the association between spatial displacement and potential influencing variables, linear regressions were calculated using a mixed effects model to account for repeat measurements (multiple maps) in several cases. For regression analyses, maps of the great cardiac vein and aorta were excluded. Demographic parameters, antiarrhythmic medication, mapped cardiac chamber, left-ventricular ejection fraction, origin of PVCs and median coupling interval were defined as fixed effects. Patient-ID was defined as random effect. The regression coefficient calculated in the linear mixed effects model was used to determine the alteration of spatial displacement depending on the change of the predictive variable. Two-sided $p<0.05$ were considered statistically significant. The reported $p$-values are used as descriptive measures only. All statistical calculations were performed in IBM SPSS Version 26.0.0.0.

\section{Results}

Baseline parameters are shown in Table 1. Twenty-two patients were included in the study (55\% male, age $63 \pm 16$ years). Twenty-four ablation procedures were performed using hybrid mapping. In two cases, a second ablation procedure was performed: One patient with cardiac sarcoidosis developed PVCs of a different morphology that was treated via catheter ablation 9 months after the first procedure. Another patient showed PVCs originating close to the His-bundle. After careful RFC-application and initial suppression of PVC in the first procedure, early recurrence of the targeted PVCs necessitated a second catheter ablation, which resulted in lasting suppression of PVCs. In total, 40 three-dimensional maps containing 5798 hybrid points were analyzed. An example for the impact of the novel mapping modality is shown in Fig. 1. Twenty-four maps (60\%) were recorded in the left ventricle (LV), 12 maps (30\%) in the right ventricle (RV), 2 maps (5\%) in the great cardiac vein and 2 maps (5\%) in the proximal aorta. In 12 procedures (50\%), the origin of the mapped PVC-morphology was confirmed in the ventricular outflowtracts (6 RVOT, 6 LVOT, outflow-tract PVCs). Non-outflow tract PVCs originated from the LV in 9 cases 
(39\%; LV-summit: 3, inferoseptal LV: 2, free LV-wall: 1, posteromedial papillary muscle: 1, posterior mitral annulus: 1, septal LV: 1 ) and from the basal RV in 1 case. Two patients (8\%) showed PVCs with an epicardial origin, which were treated by ablation via the great cardiac vein. Mean correction for spatial displacement was $3.8 \pm 1.5 \mathrm{~mm}$ for all mapping points. Median PVC-coupling interval was $552 \mathrm{~ms}$ (IQR $83 \mathrm{~ms})$. 
Table 1

Baseline parameters

\begin{tabular}{|ll|}
\hline Characteristics & Total (n = 22) \\
\hline Sex & $12(55 \%)$ \\
\hline - male & \\
\hline Age & $63 \pm 16$ years \\
\hline Structural heart disease & $13(59 \%)$ \\
• coronary artery disease & $9(41 \%)$ \\
- dilated cardiomyopathy & $2(9 \%)$ \\
- cardiac sarcoidosis & $1(5 \%)$ \\
- history of myocarditis & $1(5 \%)$ \\
\hline Impaired ejection fraction & $12(55 \%)$ \\
- mild & $6(27 \%)$ \\
- moderate & $4(18 \%)$ \\
- severe & $2(9 \%)$ \\
\hline PVC-burden in 24h-Holter & $26.2 \pm 15.8 \%$ \\
\hline Clinical Symptoms & $14(64 \%)$ \\
\hline - dyspnea & $14(64 \%)$ \\
- palpitations & $1(5 \%)$ \\
\hline - syncope & \\
\hline Cardiovascular risk factors & $12(55 \%)$ \\
• hypertension & $4(18 \%)$ \\
- diabetes mellitus & $7(32 \%)$ \\
- chronic kidney disease & $5(23 \%)$ \\
- prior myocardial infarction & \\
\hline BMl & $27.4 \pm 4.5$ \\
\hline Prior ventricular ablation & $3(15 \%)$ \\
\hline Antiarrhythmic Medication & $1(5 \%)$ \\
\hline
\end{tabular}


Categorial and ordinal parameters are displayed as absolute number, relative proportion in parentheses. Continuous parameters are shown as mean \pm standard deviation.

On average, the spatial displacement of mapping points was $1.3 \pm 0.4 \mathrm{~mm}$ larger for non-outflow-tract PVCs ( $4.5 \pm 1.5 \mathrm{~mm}$ vs. $3.2 \pm 1.2 \mathrm{~mm} ; \mathrm{p}=0.028$ in linear mixed effects model). The impact of the site of PVC-origin on spatial displacement is shown in Fig. 2. A trend towards larger displacement values with longer PVC coupling interval was observed $(p=0.068)$. Patient age, BMI, sex, antiarrhythmic medication, left-ventricular ejection fraction and the mapped cardiac chamber had no influence on spatial displacement (Table 2).

Table 2

Influence of predictive variables on median spatial displacement

\begin{tabular}{|llll|}
\hline Variable & Regression coefficient $(\mathrm{mm})$ & $\mathbf{9 5 \%} \mathbf{C l}$ & P-value \\
\hline Age (per 10 years increase) & -0.14 & $(-0.77,0.50)$ & 0.683 \\
\hline BMI (per point increase) & 0.06 & $(-0.15,0.27)$ & 0.638 \\
\hline Sex & & & 0.363 \\
\hline Left-ventricular ejection fraction & & & 0.917 \\
\hline Antiarrhythmic medication & & & 0.836 \\
\hline Coupling interval (per 100ms increase) & 0.65 & & 0.068 \\
\hline $\begin{array}{l}\text { Area of PVC-origin } \\
\text { (outflow-tract vs. non-outflow-tract) }\end{array}$ & & & $0.028^{*}$ \\
\hline Mapped chamber & & & 0.708 \\
\hline
\end{tabular}

Predictive variables and their respective influence on median spatial displacement. For continuous variables, the linear regression coefficient and the $95 \%$ confidence interval is displayed. We observed a significant influence for the area of PVC-origin.

\section{Discussion}

For catheter ablation of PVCs, accurate mapping is essential for correct localization of ablation targets and efficient suppression of PVCs. Even small inaccuracies in mapping might lead to unsuccessful ablation attempts, which may induce edema in the myocardial area of interest and complicate further ablation attempts [10]. The main finding of this study was that the spatial displacement of mapping points, determined by movement of the catheter tip during PVC compared to the preceding sinus beat, is larger in activation mapping of non-outflow tract PVCs compared to outflow tract PVCs. This has implications for mapping procedures targeting e.g. the Purkinje system and the LV wall including papillary muscles. Hybrid activation mapping is a novel tool to correct for spatial displacement of the mapping catheter due to asymmetrical contraction of myocardium during premature ventricular 
contractions (PVC). First clinical results have been published [8], but the extent of spatial displacement and influencing factors remain unclear.

Our results show that the average mapping point is displaced by ca. $4 \mathrm{~mm}$, with a $33 \%$ larger displacement for non-outflow tract PVCs. The extent of spatial displacement observed in our study is supported by the observation by Steyers et al [9]. Andreu et al. described a median spatial displacement of $9.42 \mathrm{~mm}$ (IQR 6.19-12.85) in a study including 55 patients, analyzing 6923 mapping points [7]. In this study, the authors manually corrected for the shift by re-annotating each point to the location of its preceding sinus rhythm beat. De Potter et al. found a mean spatial displacement of $8.9 \pm 5.5 \mathrm{~mm}$ in a small sample of 127 hybrid points using automatic correction for spatial displacement [8]. Differences in origin of the PVCs and potentially of coupling intervals reported in that study might be a reason for the difference in the reported displacement values.

The cranial portions of the heart containing the outflow tracts and the valvular plane are more fixed by adjoining structures like the great vessels in comparison to non-outflow-tract myocardium, which is surrounded by the pericardial space [7] and physiological displacement is less prominent than in the apical portions or the free wall of the ventricles. This might be a factor limiting the extent of spatial displacement in PVCs originating from the ventricular outflow tracts, especially in patients with preserved ventricular function. Additionally, parts of the outflow tracts are located close to the AV-node and Hisbundle. Ectopic electrical activity might therefore enter His-purkinje-system earlier than in non-outflow tract PVCs. The resulting myocardial contraction might resemble the physiological contraction more closely, resulting in limited spatial displacement. Similarly, small differences of spatial displacement between mapping points located in different myocardial areas were observed in an earlier study [7]. However, the authors differentiated between location of mapping points, while we differentiated between different areas of PVC-origin. The aforementioned anatomical conditions for the outflow tracts might play a role for both findings. However, an earlier entry of electrical activity into the His-Purkinje-system is influenced by PVC-origin, not on the sole location of mapping points. Therefore, the results are not entirely comparable.

Reported data concerning influencing factors for spatial displacement are heterogeneous:

While Steyers et al. reported no impact for PVC-coupling interval on spatial displacement, Andreu et al. observed a significantly larger displacement for mapping points with a lower coupling interval $[7,9]$. In contrast, we saw a trend towards larger displacement for PVCs with a longer coupling interval. The difference in the reported results could be explained by disparities in the patient collectives: The vast majority of patients in the study of Andreu et al. had PVCs originating from the ventricular outflow tracts [7], while the origin of PVCs was distributed almost equally between ventricular outflow tracts and nonoutflow tract myocardium in our study population. Since the ventricular outflow tracts are more fixed by adjoining vessels, inadequate ventricular filling associated with a shorter coupling interval might be more relevant for spatial displacement for PVCs originating in the cranial portions of the heart [7]. 
The previously reported influence of the mapped heart chamber on spatial displacement [7] could neither be confirmed by our study nor by Steyers et al. [9]. Again, this discrepancy might be explained by differences in the study populations: PVCs originating outside the outflow tracts are associated with structural heart disease [11], which was more prevalent in the left ventricle in our study population. That these PVCs showed significantly more displacement, might balance out the association between spatial displacement and mapped chamber previously reported for PVCs originating around the valvular plane [7].

Although first clinical results show the efficacy of catheter ablation using hybrid mapping [8, 9, 12], a clinical benefit compared to conventional activation mapping is yet to be demonstrated. Since standard ablation catheters have a 3- to 4-mm tip, it seems plausible that a correction for spatial displacement might improve the accuracy of RFC-delivery in a clinically significant extent. According to our results this might be especially relevant for non-outflow tract PVCs, as those showed a significantly larger displacement. With broader use of the novel mapping software, we can expect to learn more about the spatial displacement of mapping points in PVCs. A randomized, controlled study comparing results of ablation procedures with hybrid activation mapping against procedures with conventional activation mapping would be desirable in order to evaluate the potential clinical benefit of the novel mapping feature.

\section{Limitations}

In some maps, singular points with a very high value for spatial displacement were observed. Since even small catheter displacement is supposed to be recorded, stability filters are disabled during hybrid mapping. Therefore, movement of the catheter between PVC and the preceding sinus beat may lead to false-high values for spatial displacement. Although we carefully checked all annotations during the procedure, singular false-high measurements for spatial displacement cannot be completely ruled out. Since we used median values for our analyses, we believe this bias to be of minor relevance for our results.

Secondly, even though our analysis covered a total of 5798 individual mapping points, the studied patient population of 22 constitutes a small sample size. Thirdly, all analyzed data was recorded using the CARTO system. Although the described spatial displacement should be detectable irrespective of the mapping system, small differences between the platforms cannot be ruled out.

\section{Conclusion}

Ectopic activation of the ventricular myocardium during PVCs results in a spatial displacement of mapping points. We observed a mean spatial displacement of $3.8 \pm 1.5 \mathrm{~mm}$ that was dependent on the location of PVC-origin: mapping points of PVCs with non-outflow-tract origin showed a larger displacement than PVCs originating from the outflow tracts. The correction for spatial displacement may 
help to improve accuracy of RFC-delivery in catheter ablation of PVCs. Our results suggest that this is especially relevant for PVCs with non-outflow-tract origin.

\section{Declarations}

\section{Compliance with ethical standards}

In our retrospective study, all patients consented to the scientific use of data acquired during routine procedure. All data was analyzed anonymously. No ethical board approval was required.

\section{Consent for publication}

Not applicable

\section{Availability of data and materials}

All data and materials are available from the corresponding author upon request.

\section{Competing interests}

AR received travel grants from Biosense, Medtronic, St. Jude Medical, Cardiofocus, EP Solutions and Ablamap and lecture and consultant fees from St. Jude Medical, Medtronic, Biosense, Cardiofocus, Novartis and Boehringer Ingelheim.

$\mathrm{CM}$ received compensation for participation as consultant for Biosense Webster, Boston Scientific. Speaker for Abbott.

AM received speaker's honoraria and travel grants from Medtronic, Biosense Webster, Bayer, Boehringer Ingelheim and Cardiofocus.

\section{Funding}

This research did not receive any specific grants from funding agencies in the public, commercial, or notfor-profit sectors.

\section{Authors' contributions}

MN drafted the work. CM and MN were responsible for the work's conception. RS, PK, CM substantively revised the work. All other authors were involved in the acquisition of data. All authors read and approved the final manuscript.

\section{Acknowledgements}

We would like to thank Alexandra Höller (Institute for Medical Biometry and Epidemiology, University Hospital Hamburg) for her counsel during statistical analysis and Julia Weinert (Biosense Webster) for 
her help with the visualization of mapping data.

\section{References}

1. Fichtner, S., et al., Safety and efficacy in ablation of premature ventricular contraction: data from the German ablation registry. Clin Res Cardiol, 2017. 106(1): p. 49-57.

2. Schleberger, R., et al., Outcome of catheter ablation of non-reentrant ventricular arrhythmias in patients with and without structural heart disease. Eur J Med Res, 2020. 25(1): p. 4.

3. Bogun, F., et al., Radiofrequency ablation of frequent, idiopathic premature ventricular complexes: comparison with a control group without intervention. Heart Rhythm, 2007. 4(7): p. 863-7.

4. Latchamsetty, R., et al., Multicenter Outcomes for Catheter Ablation of Idiopathic Premature Ventricular Complexes. JACC Clin Electrophysiol, 2015. 1(3): p. 116-123.

5. Cronin, E.M., et al., 2019 HRS/EHRA/APHRS/LAHRS expert consensus statement on catheter ablation of ventricular arrhythmias: executive summary. Europace, 2020. 22(3): p. 450-495.

6. Azegami, K., et al., Spatial resolution of pacemapping and activation mapping in patients with idiopathic right ventricular outflow tract tachycardia. J Cardiovasc Electrophysiol, 2005. 16(8): p. 823-9.

7. Andreu, D., et al., Displacement of the target ablation site and ventricles during premature ventricular contractions: relevance for radiofrequency catheter ablation. Heart Rhythm, 2012. 9(7): p. 1050-7.

8. De Potter, T., et al., Premature ventricular contractions cause a position shift in 3D mapping systems: analysis, quantification, and correction by hybrid activation mapping. Europace, 2020. 22(4): p. 607-612.

9. Steyers, C.M., 3rd, et al., Ablation using 3D maps adjusted for spatial displacement of premature ventricular complexes relative to sinus beats: Improving precision by correcting for the shift. J Cardiovasc Electrophysiol, 2019. 30(11): p. 2319-2325.

10. Baser, K., et al., Predictors of outcome after catheter ablation of premature ventricular complexes. J Cardiovasc Electrophysiol, 2014. 25(6): p. 597-601.

11. Marcus, G.M., Evaluation and Management of Premature Ventricular Complexes. Circulation, 2020. 141(17): p. 1404-1418.

12. Compagnucci, P., et al., Recent advances in three-dimensional electroanatomical mapping guidance for the ablation of complex atrial and ventricular arrhythmias. J Interv Card Electrophysiol, 2020.

\section{Figures}




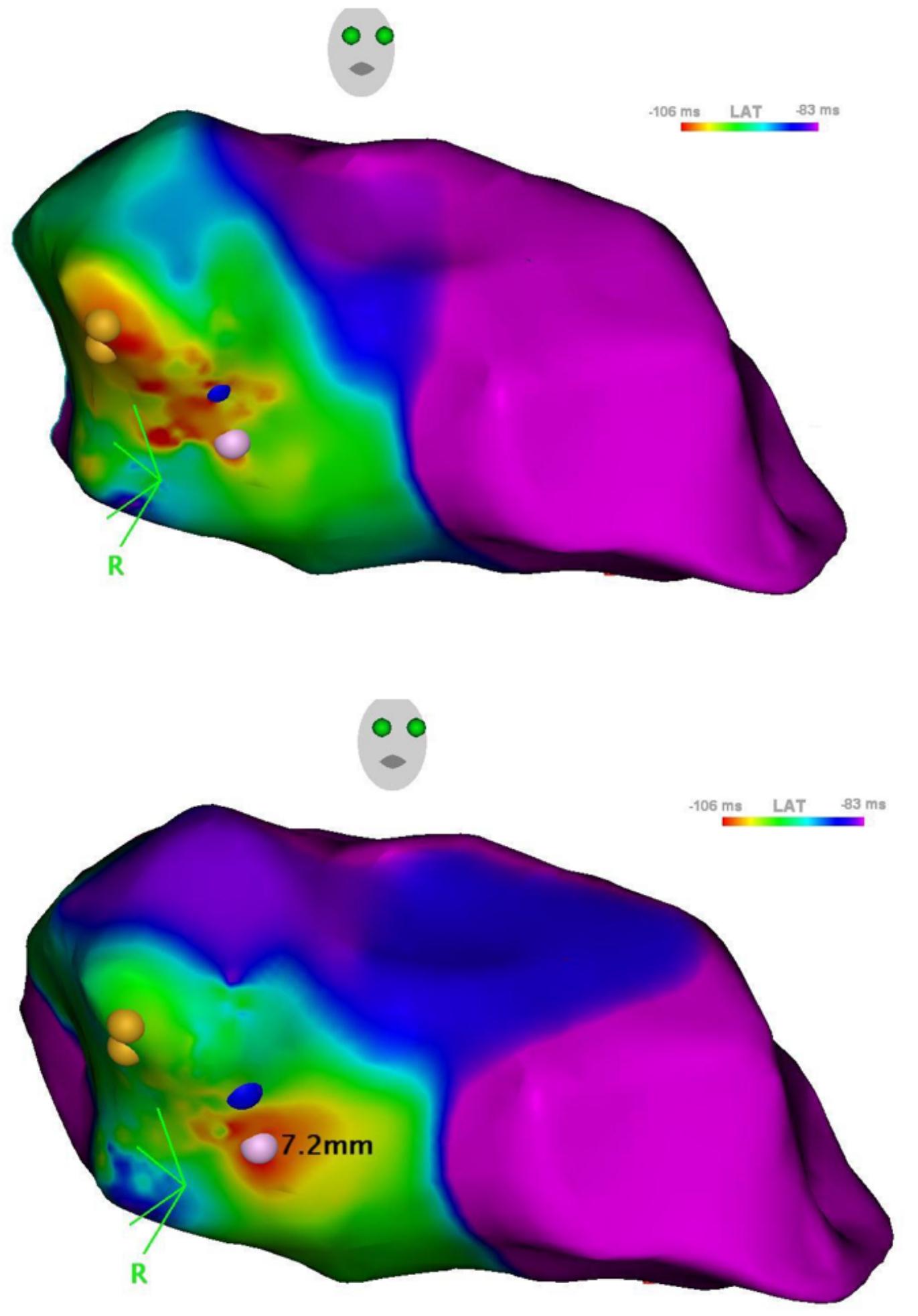

\section{Figure 1}

Visualization of spatial displacement. Activation maps of PVCs using CARTO III, Software Version 7 Carto Prime, Biosense Webster, RAO-view $\left(45^{\circ}\right)$. Upper panel: Conventional activation map of a left ventricle during an ablation procedure of PVCs with left-ventricular origin. Lower panel: Activation map with correction for spatial displacement for the same PVC-morphology. Blue point = point with earliest local activation time (LAT) in conventional map; rose point = point with earliest LAT for hybrid map; yellow 
points $=$ His-potentials. Mapping colors indicate the local activation time relative to the selected reference in surface ECG. Red areas show the earliest activation, while late activation is visualized as purple area (see LAT scale in the top right corner). In this case, the area of earliest local activation was spatially displaced by $7.2 \mathrm{~mm}$ compared to the conventional activation map. The conventional map suggested a focus closer to the His-bundle. Correction for spatial displacement revealed the correct target for successful ablation in an area associated with a lower risk for lesion of the specific conduction system.

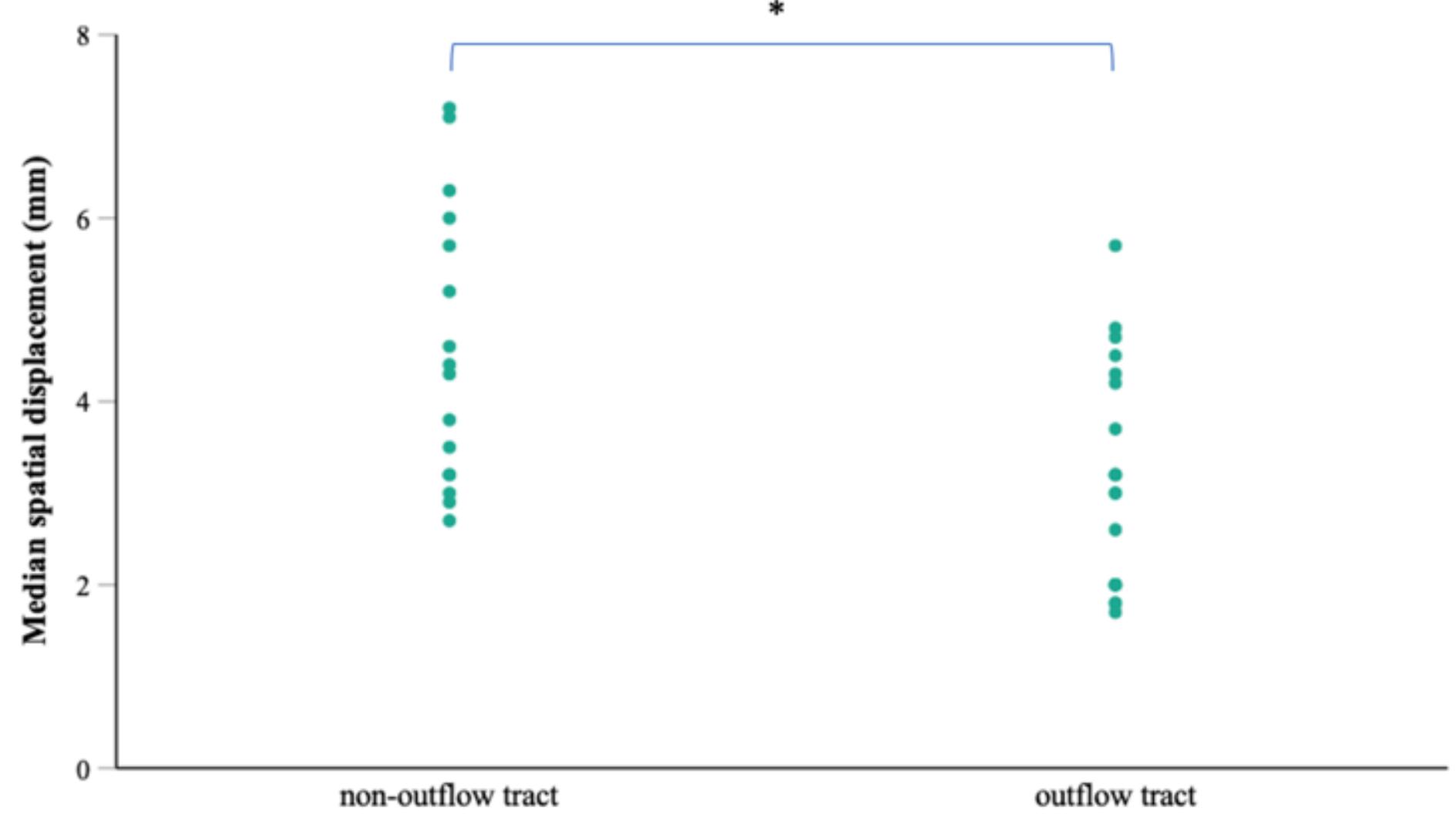

\section{Area of origin}

\section{Figure 2}

Influence of PVC-origin on spatial displacement. Scatter plot showing spatial displacement of mapping points depending on the area of PVC-origin. The median spatial displacement was significantly lower in maps recorded for PVCs originating in the ventricular outflow tracts $(p=0.028)$. Standard deviation was comparable in both groups. 